\title{
Indoor Localization System Using Wi-Fi Technology
}

\author{
Noor Abdul Khaleq Zghair ${ }^{1}$, Muayad Sadik Croock², Ali Abdul Razzaq Taresh ${ }^{3}$ \\ ${ }^{1}$ Computer Engineering Department, University of Technology, Baghdad, Iraq. \\ ${ }^{2}$ Computer Engineering Department, University of Technology, Baghdad, Iraq. \\ ${ }^{3}$ Computer Engineering Department, University of Technology, Baghdad, Iraq. \\ nooriteng2@gmail.com,muayadkrook@yahoo.com,alifiter84@yahoo.com
}

\begin{abstract}
Recently, indoor localization has witnessed an increase in interest, due to the potential wide range of using in different applications, such as Internet of Things (IoT). It is also providing a solution for the absence of Global Positioning System (GPS) signals inside buildings. Different techniques have been used for performing the indoor localization, such as sensors and wireless technologies. In this paper, an indoor localization and object tracking system is proposed based on WiFi transmission technique. It is done by distributing different WiFi sources around the building to read the data of the tracked objects. This is to measure the distance between the WiFi receiver and the object to allocate and track it efficiently. The test results show that the proposed system is working in an efficient way with low cost.
\end{abstract}

Index Terms - Indoor Localization, Wi-Fi, tracking object, Distance measurement.

\section{INTRODUCTION}

Localization and tracking of objects have become a very important entity for the society for a long time. Nowadays, much outdoor localization and tracking solutions exist, based on Global Positioning System (GPS), which can take us within meters of certainty when locating the position of a building [1]. In indoor environments, the received GPS signals are too weak to provide an accurate object location. At present, buildings are equipped with Wireless Local Area Network (WLAN) access points, such as airports, supermarkets, hospitals, museums, etc. It can be effectively implemented to use these access points as a source and to receive WiFi signals to locate user location in these internal environments [2]. However, when it comes to localization of objects indoors, here we need to know more accurate information about the position of such objects inside a building [1].

The wide use of smart phones and other wireless devices eases the localization and tracking of such devices, which in turn allocates the position of the user. In the last couple of years, the research area has been provided with many services, including indoor localization. Indoor localization is the process of obtaining a device or user location in an indoor setting or surrounding. The localization of the indoor devices has been extensively studied, especially in the industrial environment and for wireless sensor networks and robots [3]. In the previous few years, localization of mobile phones has been a real necessity for which an assortment of offered technologies has been used in order to obtain it with good accuracy. Most of the existing systems that offer indoor localization services used different wireless technologies, such as Bluetooth, WiFi, signals of cellular towers and ZigBee. The methods using Wi-Fi are more popular because WiFi networks dominate most public buildings, with no need for additional infrastructure and without limitations on the number of items to be monitored or tracked [4].

There are various indoor localization algorithms based on wireless signals e.g. ([5], [6], [7], [8] and [9]). The main challenges in using these algorithms are to provide acceptable 
accuracy and deployment cost. For these reasons, a lot of interest has been gained in many research communities using WiFi for indoor localization techniques [10].

Therefore, the proposed method of this paper uses WiFi signals strength to compute the distance between the user's device and the WiFi sender (Access Points (AP) or router). On the other hand, the problem is that WiFi signals are changing as the obstacles, such as walls, equipment found in the building, and the people around it can affect the signal strengths [4]. The proposed indoor localization technology based on WiFi signal solves this problem by distributing the access point to guarantee the coverage of the whole building. It is simple in implementation and estimation and it can localize the position of a user's device on each floor.

\section{RELATED WORKS}

There are different techniques used in indoor localization and some of them have been referenced in this section. In addition, several solutions are provided based on wireless technologies, such as Bluetooth, ZigBee, signals of cellular towers, WiFi and RFID. The related works can be categorized into two subsections.

Indoor localization can be defined as any system that provides the exact location of objects (a personal item) in a closed structure, such as supermarkets, hospitals, universities, airports and subways [11]. Indoor location system has become very popular in recent years. Therefore, many systems and technologies have been proposed to get the user and device localization based into Object. The object can be a mobile phone, keys with attached microcontrollers a keychain, a wallet with embedded microcontroller or microcontroller in a child pocket, among others [12].

One of the existing solutions for indoor localization was provided in [13] to discuss various indoor localization systems that are proposed in the literature and show challenges, such as accuracy of the localization systems. While the authors in [3] introduced an extensive study of many indoor localization techniques, such as the Angle of Arrival (AoA), Flight Time (ToF), Return Time of Flight (RTOF), the Received Signal Strength (RSS), Ultra-wide band (UWB), Radio Frequency Identification of Device (RFID), Bluetooth and Systems. The study looks at the localization and positioning of people users and their devices.

For wireless indoor location, various wireless technologies are used. One of the existing research work based on Wi-Fi was indoor triangulation system [14]. This system uses its own Wi-Fi nodes to measure the location of several Wi-Fi devices within the indoor area. The solution provided is required for its own Wi-Fi node infrastructure. Localization accuracy was defined by its location and its concentration. The next notable example of WiFi localization systems is based on several radio beacons [15]. These beacons may be access points for wireless LANs, fixed Bluetooth stations and GSM towers. They used all protocols, which resulted in the unique or semi-unique identification number for beacons. By using this identifier, the lab system detects the indoor position of the user. In [16], the applications of indoor localization have improved the sensing ability to detect the current state of mobile devices. The application was introduced for smartphones and it includes offline and online levels of fingerprinting. While in [10], the authors have created an application that describes the Wi-Fi trilateration method for internal positioning using Android based mobile devices. This was done by receiving a signal strength measurement group that improves localization accuracy. Finally, in [17] the authors presented the latest developed systems or solutions and their algorithms for wireless localization on indoor 
application. In this survey, the author presented existing wireless solutions for indoor positioning and sought to classify different methods and systems.

\section{PROPOSED SYSTEM DESIGN}

The result of the localization process is the future location of the users, which are obtained by using a specific localization technique [10]. The previous methods are based on knowledge of reference point positions to Wi-Fi and Wi-Fi ID for each person. In this paper the access point is used, which broadcasts Wi-Fi signal, and the person phone for presuming to have the Wi-Fi connection capabilities that automatically connects to the access point in the building. The result of the system is shown in the interface of an indoor localization system that runs in the server as shown in Fig. 1. The system is based on the connection between the phone and access point to identify the person and related location in allocated floor of the considered building.

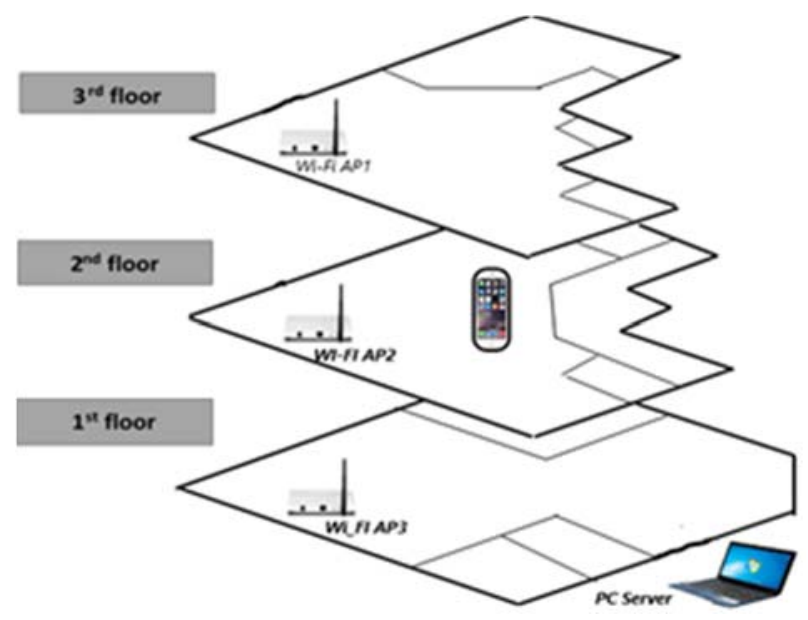

FIG. 1. THE INDOOR LOCALIZATION SYSTEM.

The proposed system consists of two main parts. The first part is the input stage that includes ESP8266 Arduino to detect Wi-Fi signal. In this part, the designed system is simulated using MATLAB Graphical User Interface (GUI) to display the processed data. The two stages are designed independently before being joined into one system. This is to ensure that if there are any errors, they are independently and automatically considered and corrected. On the other hand, the other part is the user's device, which has a Wi-Fi facility such as: smart-phones, PDAs, etc. This section is divided into two subsections as follows:

\section{A. Description of the Wi-Fi System Architecture and Program}

In this system, a microcontroller is used (node MCU 1.0) and the Arduino. These components are adopted as they are easy in programming and use. In addition, they are cheap comparing to other controllers and easy in implementation. Finally, the design of the system interface is performed, in which it becomes simple with the lowest cost possible. The components of the system have been explained as follows:

\section{ESP8266 Arduino}

The ESP8266 Arduino compatible module is a cheap Wi-Fi chip with full TCP / IP capabilities. This small module allows microcontrollers to connect to the Wi-Fi network and establishe simple TCP / IP connections using Hayes style commands [18]. ESP8266 Arduino is shown Fig. 2. 


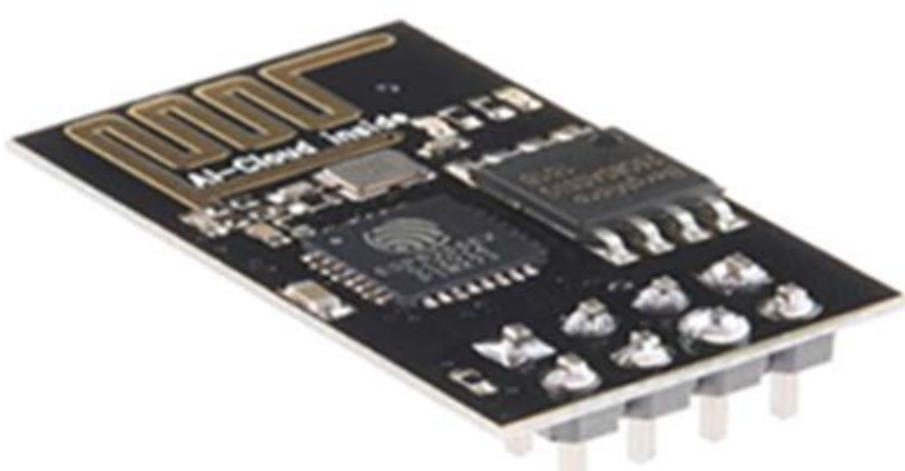

FIG. 2. ESP8266 ARDUINO

In this paper, the ESP8266 Arduino is used to connect the server (PC) and the three-access point to detect the Wi-Fi signal. The Arduino is chosen instead of Wi-Fi shield because it is cheaper than Wi-Fi shield, easy in implementation, and easy in programming. ESP8266 is built in with node MCU, so we don't have to buy two boards for interconnection and we avoid connections problems.

\section{Node MCU 1.0}

Node MCU is a firmware on ESP8266 and it is basically a System on Chip (SoC). In addition, the node MCU is an IoT open source platform. By default, the word "MCU node" refers to the firmware rather than development kits [18]. Node MCU is smaller than Arduino board, and also it has a built in ESP8266, thus we don't have to connect external ESP8266 for easy implementation as shown in Fig.s 3 and 4.

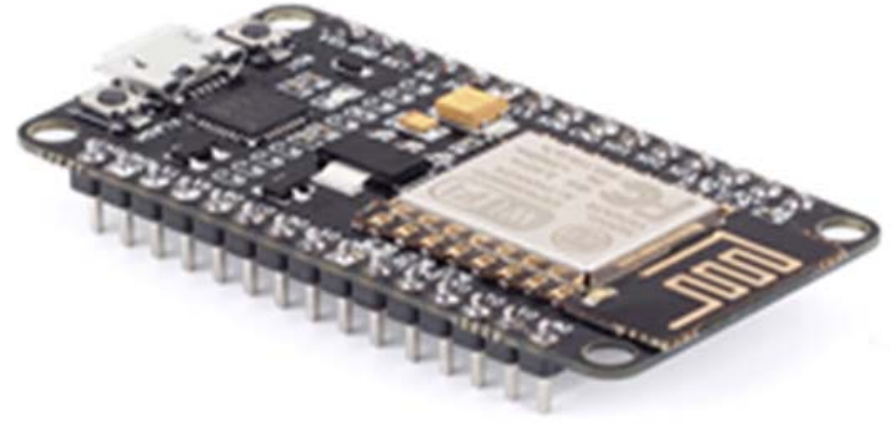

FIG. 3. NODE MCU (1.0)

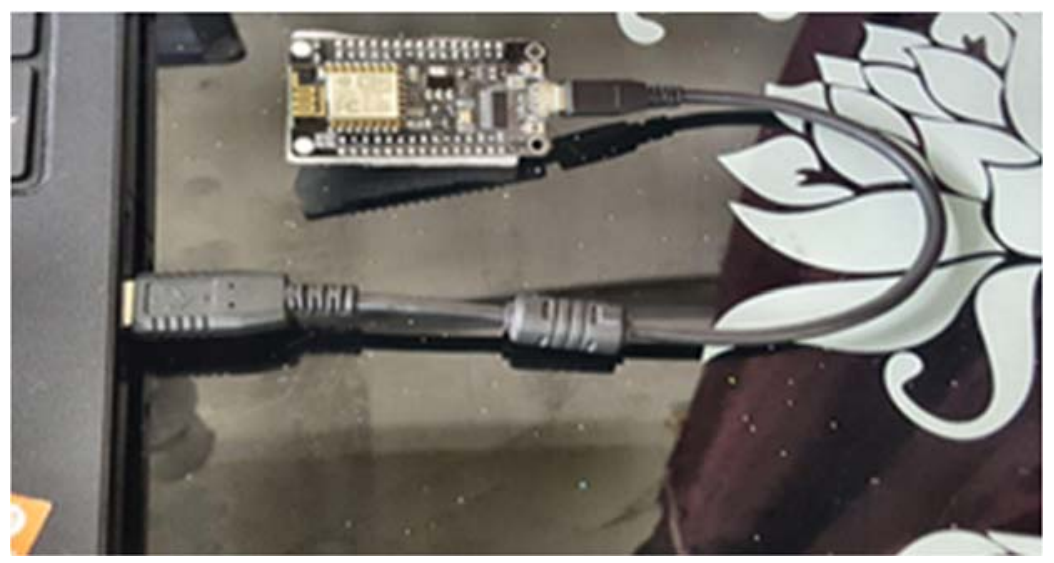

FIG. 4. CONNECT THE NODE MCU 1.0 TO THE USB PORT 
The node's MCU can be powered by a USB connection or an external power supply. The MCU node software includes a series monitor, which allows text data exchange with the board. The node MCU provides access to the GPIO (General Purpose Input/Output) and access to development. Fig. 5; shows the pin mapping from the API documentation [18].

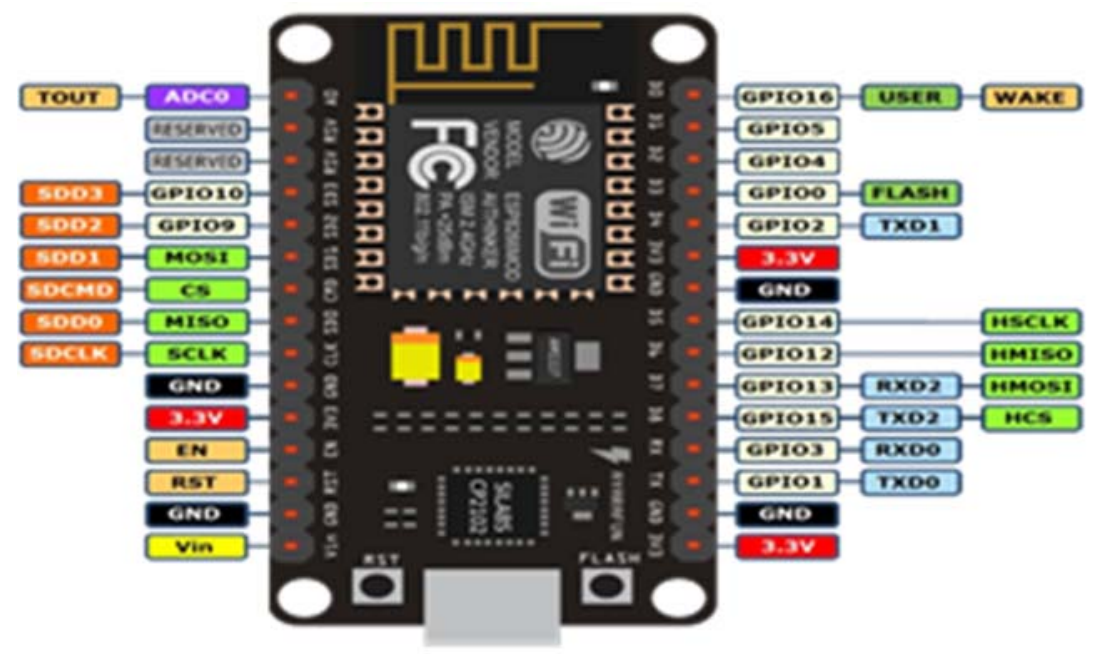

Fig. 5. PINS OF NODE MCU CONNECTED TO EsP8266 [18]

\section{B. The proposed Algorithm}

The operation of the proposed algorithm is built on request to know a location of a device inside the building. The system is performing a search over the considered access points to find out the location of the requested device. This search can be done by inserting the floor number in the input text that is labeled (port name) in GUI, then click on the start button to setup the access point of the detect floor to detect and track data (Wi-Fi signals) from the port in the location. The obtained results including the data and the time of the user devices that connect to this access point are shown in the screen of GUI. Fig. 6 depicts the flowchart of the proposed algorithm for the indoor localization system. First, the system is doing configuration to all access points and reading any connection request. After that, it gives an IP address to each phone connected while reading its MAC address. In the next step, the system performs a comparison between the result value of MAC address and the stored data in the system. If the result is equal, the system assumed that the person is registered (employee). Otherwise, this person is not registered (not an employee). Finally, the system makes a loop to update person position changes. The result of the test is depending on the distance that is estimated by the strength of the signal which is presented as a circle with a radius around the access point. 


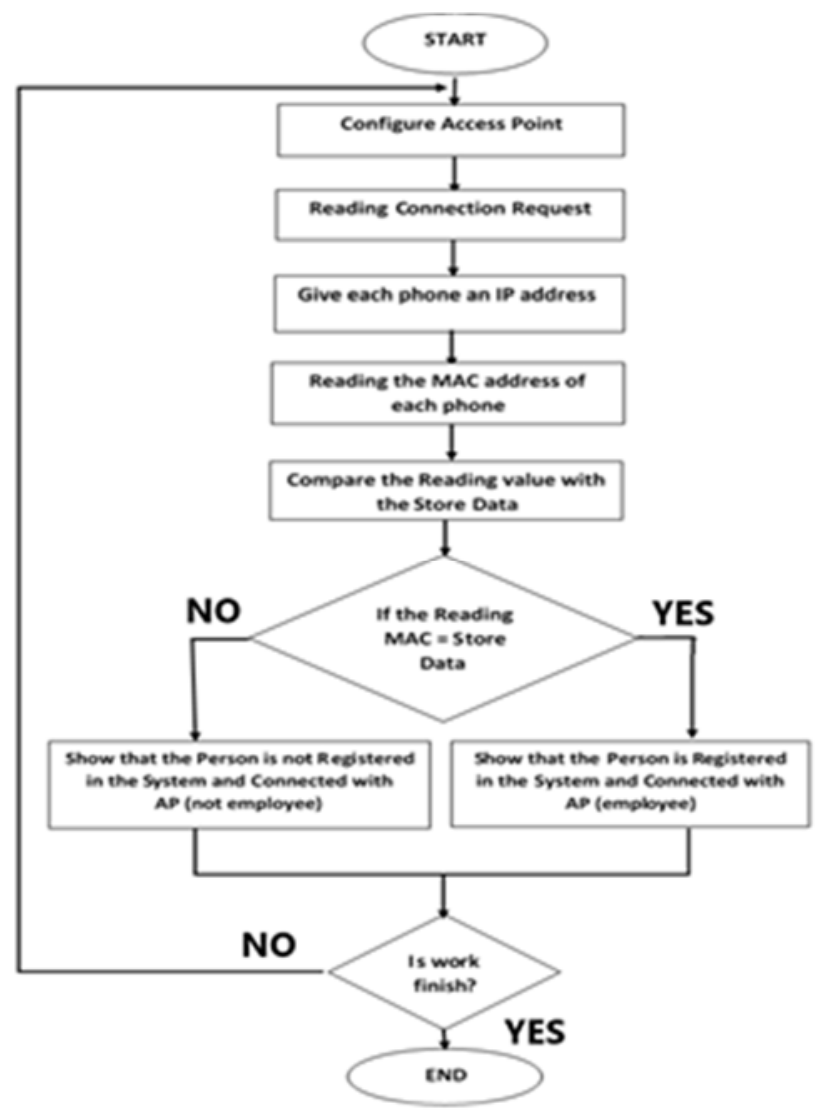

FIG. 6. INDOOR LOCALIZATION PROPOSED ALGORITHM

\section{GUI Design}

Graphical User Interface (GUI) designs have one or more windows with controls, called components that enable the user to perform the interactive functions to manipulate the required control. In this paper, the microcontroller uses a program with a designed GUI using C\# programming language. The designed interface shows a window that contains a text input labeled port name to determine which device (Wi-fi Access Points) is selected to read data from. For example, the system assumes that the building contains many floors and you want to choose a floor to track an object using the WiFi signals received by sources (access points). The start button is used to begin the setup of the selected device, wanted to be detected and tracked using Wi-Fi signals. The designed GUI also contains a text output labeled time to show the result of the searching amongst the WiFi devices connected to the specific source in an allocation. In addition, the GUI includes time connected with the port to show the data and the time the data received from the port, Fig. 7 shows the designed GUI. 


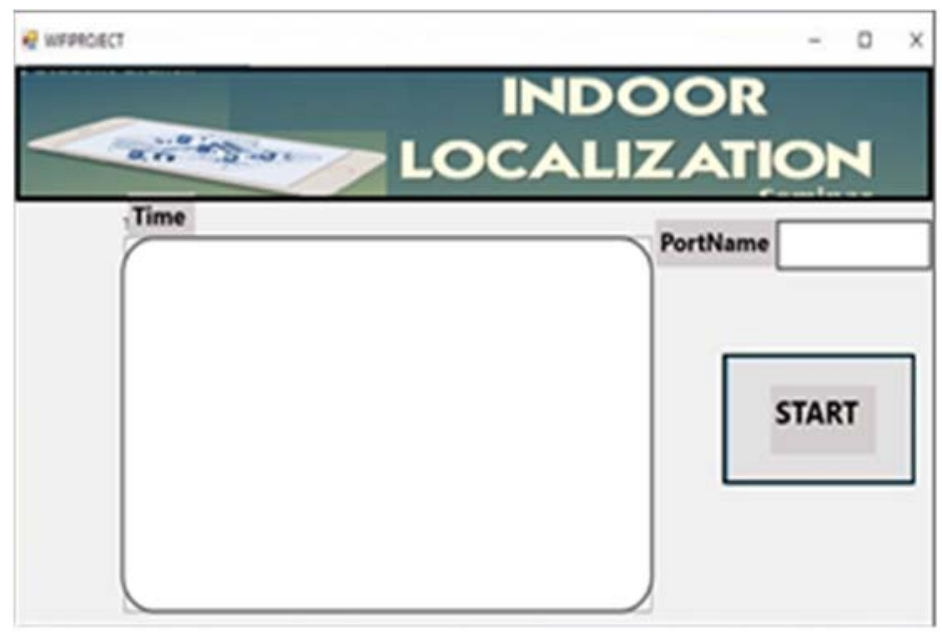

FIG. 7. DESIGNED GUI

\section{The User Device}

The user or client device must be supposed to have Wi-Fi capabilities automatically which are automatically turned on to the Wi-fi when such device enters the considered building. In this project, the smartphones are chosen as they have a Wi-Fi and related ID to allocate them. These devices are selected due to availability and ability of detecting Wi-Fi stations and establishing the connection based on the higher strength of the station. The WiFi ID is used to detect the current location of such user based on the provided Media Access Control (MAC) address. The MAC address is used for two reasons. The first one is that MAC addresses are unique identifiers, while the second reason is related to the node MCU that gives IP address automatically to any connected device. This IP is changed to the same phone depending on the connection of the user. Therefore, it can be adopted for the purpose of this paper.

\section{THE RESULT}

In this project, the test environment is selected to be the building of the computer engineering department at University of Technology. This is done by allocating an access point to each floor of three. When the user (employee) enters the building, his phone is connected automatically to the access point in the first floor. The system reads his name and knows its location. At the time of going to the second floor, his smartphone is disconnected from the access point of the first floor and connects to access point of the second floor automatically. This is because the signal of the second floor is stronger than the first-floor signal. Therefore, the proposed system reads his signal and location from the other access to the related floor. Fig. 8 shows the results at the moment of connecting a device. In this figure, it is seen that the number of connections is two devices. The first device is with the MAC address of (ec:1f:72:8f:9c:7f) and this person is an employee (the MAC = stored data). While the second device is with MAC address of (ec:1f:72:61:37:2d) and this one is an employee too. Finally, it is noted in this figure that the results or searches can be updated at any time for changing a person position in the floors. On the other hand, Fig. 9 shows the results when no devices are connected in this floor. 


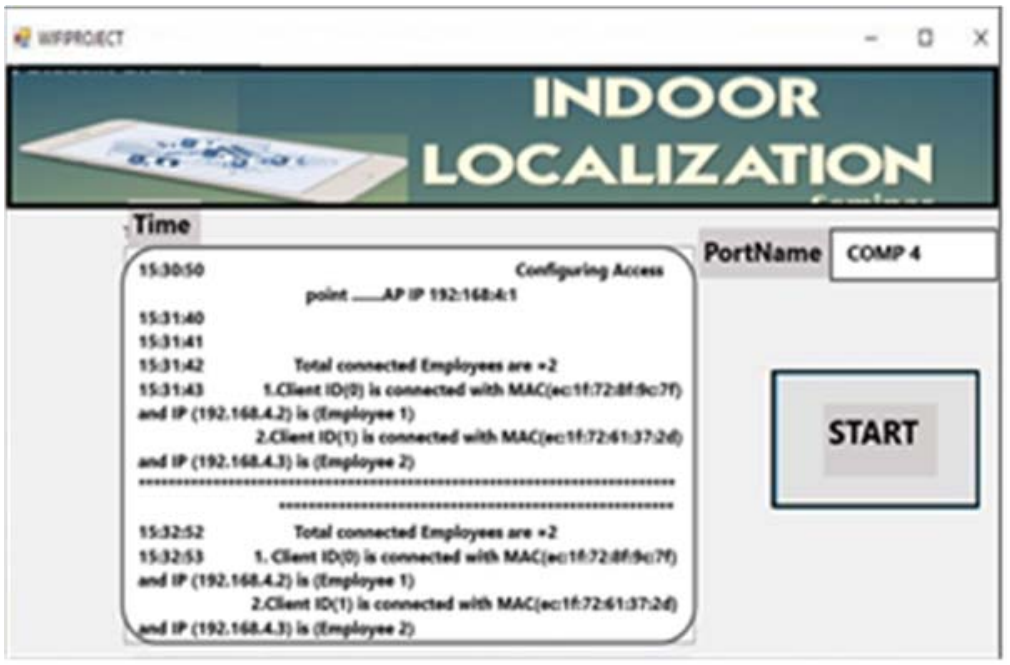

Fig. 8. REAding Wi-Fi SIGNALS FROM the ACCESS POINT (CONNECTED DEVICES)

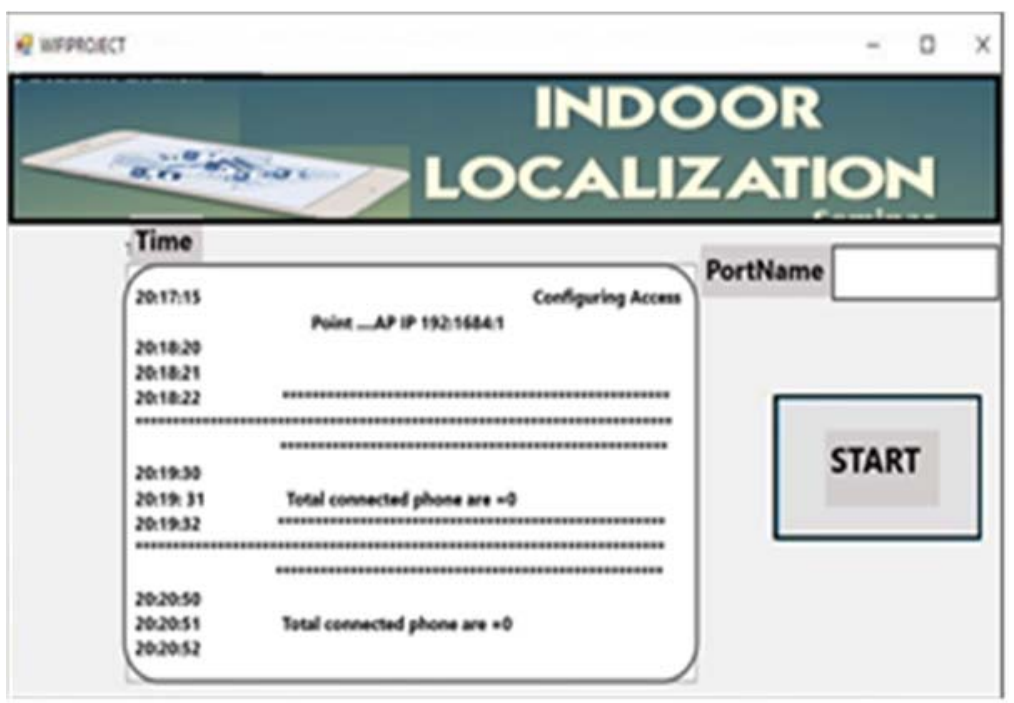

Fig. 9. REAding Wi-Fi SigNALS FROM THE ACCESS POINT (DISCONNECTED DEVICES)

\section{CONCLUSIONS AND FUTURE WORK}

In this paper, the Wi-Fi signal strength-based object detection and tracking system in the indoor environment was proposed. The proposed system used the WiFi signal strength read from the distributed access points to evaluate the position of the required object. The location is assigned using the MAC address of the smartphone of each user involved. The strength of reading WiFi signals from an access point referred to the location of such a device. The smartphone device is connected to the nearest access point based on the WiFi signal strength, and it disconnected from the far points. The obtained results showed the accurate performance of the proposed system in terms of allocating objects.

\section{REFERENCES}

[1] Marcus Utter, “Indoor Positioning using Ultra-wideband Technology”, Examensarbete 30 hp, December 2015

[2] Fr 'ed' eric Evennou and Franc, oisMarx, "Advanced Integration of Wi-Fi and Inertial Navigation Systems for Indoor Mobile Positioning”, EURASIP Journal on Applied Signal Processing, Volume 2006, Article ID 86706, Pages 1-11, DOI 10.1155/ASP/2006/86706.

[3] Faheem Zafari, Athanasios Gkelias, Kin K. Leung, “A Survey of Indoor Localization Systems and Technologies”, arXiv:1709.01015v1 [cs.NI] 4 Sep 2017. 
[4] Bianca BOBESCU, Marian ALEXANDRU, “Mobile Indoor Positioning Using Wi-Fi Localization”, Review of the Air Force Academy, No 1 (28) 2015.

[5] N. Patwari, A.O. Hero, M. Perkins, N.S. Correal, R.J. O'dea, "Relative location estimation in wireless sensor networks", IEEE Transactions on Signal Processing, 51 (8), pp. 2137-2148, 2003.

[6] M. Youssef, A. Agrawala, "The Horus WLAN Location Determination System”, in Proc. MobiSys '05 Proceedings of the 3rd international conference on Mobile systems, applications, and services, pp. 205-218, 2003.

[7] B. Cook, G. Buckberry, I. Scowcroft, J. Mitchell, T. Allen, "Location by Scene Analysis of Wi-Fi Characteristics”, London Communications Symposium, Sep. 2006.

[8] N. Kothari, B. Kannan, M. B. Dias, "Robust Indoor Localization on a Commercial Smart-Phone”, Procedia Computer Science, vol. 10, pp. 1114-1120, 2012.

[9] L.T. Nguyen, J. Zhang, "Wi-Fi fingerprinting through active learning using smartphones”, in Proc. of the 2013 ACM conference on Pervasive and ubiquitous computing adjunct publication, pp. 969-976, 2013.

[10] Maxim Shchekotov, "Indoor Localization Methods Based on Wi-Fi Lateration and Signal Strength Data Collection”, Proceeding of the 17th Conference Of Fruct Association, 2013.

[11] Zahid Farid, Rosdiadee Nordin, andMahamod Ismail, "Recent Advances in Wireless Indoor Localization Techniques and System”, Hindawi Publishing Corporation, Journal of Computer Networks and Communications, Volume 2013, Article ID 185138, 12 pages, http://dx.doi.org/10.1155/2013/185138

[12] Steve MAagi, “Tracking framework for object localization using Arduino”, University of Tartu, Faculty of Mathematics and Computer Science, May 2012.

[13] K. Al Nuaimi and H. Kamel, “A survey of indoor positioning systems and algorithms,” in Innovations in information technology (IIT), 2011, international conference on, 2011.

[14] Navizon Indoor Triangulation System Fact Sheet PDF, Web: https://www.navizon.com/files/Navizon.

[15] D. Kolsch, “The Place Lab Project”, Mobile Business Seminar, 2006.

[16] E. Martin, O. Vinyals, G. Friedland, R. Bajcsy, “Precise Indoor Localization Using Smart Phones”, in Proc. of the ACM International Conference on Multimedia, pp. 787-790, 2010.

[17] Hui Liu, Houshang Darabi, Pat Banerjee, and Jing Liu, “Survey of Wireless Indoor Positioning Techniques and Systems”, IEEE Transactions on Systems, Man, And Cybernetics_-Part C: Applications and Reviews, Vol. 37, No. 6, November 2007.

[18] Suraj Tonage , Sandhya Yemul, Rajendra Jare , Veena Patki, "IoT based home automation system using NodeMCU ESP8266 module”, International Journal of Advance Research and Development, Volume3, Issue3, 2018. 